\title{
EVERYTHING IS A CIRCLE: A NEW UNIVERSAL ORBITAL MODEL
}

\author{
Author: Aslı Pinar Tan ${ }^{\S}$
}

\begin{abstract}
Based on measured astronomical position data of heavenly objects in the Solar System and other planetary systems, all bodies in space seem to move in some kind of elliptical motion with respect to each other. According to Kepler's $1^{\text {st }}$ Law, "orbit of a planet with respect to the Sun is an ellipse, with the Sun at one of the two foci." Orbit of the Moon with respect to Earth is also distinctly elliptical, but this ellipse has a varying eccentricity as the Moon comes closer to and goes farther away from the Earth in a harmonic style along a full cycle of this ellipse. In this paper, our research results are summarized, where it is first mathematically shown that the "distance between points around any two different circles in three dimensional space" is equivalent to the "distance of points around a vector ellipse to another fixed or moving point, as in two dimensional space". What is done is equivalent to showing that bodies moving on two different circular orbits in space vector wise behave as if moving on an elliptical path with respect to each other, and virtually seeing each other as positioned at an instantaneously stationary point in space on their relative ecliptic plane, whether they are moving with the same angular velocity, or different but fixed angular velocities, or even with different and changing angular velocities with respect to their own centers of revolution. This mathematical revelation has the potential to lead to far reaching discoveries in physics, enabling more insight into forces of nature, with a formulation of a new fundamental model regarding the motions of bodies in the Universe, including the Sun, Planets, and Satellites in the Solar System and elsewhere, as well as at particle and subatomic level. Based on the demonstrated mathematical analysis, as they exhibit almost fixed elliptic orbits relative to one another over time, the assertion is made that the Sun, the Earth, and the Moon must each be revolving in their individual circular orbits of revolution in space. With this expectation, individual orbital parameters of the Sun, the Earth, and the Moon are calculated based on observed Earth to Sun and Earth to Moon distance data, also using analytical methods developed as part of this research to an approximation. This calculation and analysis process have revealed additional results aligned with observation, and this also supports our assertion that the Sun, the Earth, and the Moon must actually be revolving in individual circular orbits.
\end{abstract}

\footnotetext{
$\S$ Author: Aslı Pınar Tan

LinkedIn Website: http://www.linkedin.com/in/apinartan

E-mail: aslipinartan@superonline.com
} 


\section{ARTICLE}

Consider a system of two circles in three dimensional space, with the geometry of the system depicted as in Figure 1 in Cartesian $(\hat{\boldsymbol{x}}, \hat{\boldsymbol{y}}, \hat{\boldsymbol{z}})$ coordinates, and the defining vectors expressed in the form of generic vector Eq.s (4) - (9). Points $\mathbf{P}_{1}$ and $\mathbf{P}_{2}$ are defined on these two circles, respectively, phased apart by a constant or time $(t)$ dependent angle $\phi_{0}$ (3). Location of $\mathbf{P}_{1}$, phased at $\left[\phi_{1}(t)=\phi+\phi_{0}\right]$ (1) around its own circle, is defined by vector $\left[\overrightarrow{\boldsymbol{r}}_{1}(\phi)+\vec{\ell}(\phi)\right]$ based on Eq.s (4) - (5) and Eq. (8), and the location of $\mathbf{P}_{2}$, phased at $\left[\phi_{2}(t)=\phi\right]$ (2) around its own circle, is defined by vector $\overrightarrow{\boldsymbol{r}}_{2}(\phi)(6)$.

$$
\begin{array}{ll}
\phi_{1}(t)=\phi+\phi_{0}=\phi(t)+\phi_{0}(t)=\phi_{2}(t)+\phi_{0}(t) & \left(\text { Phase of } \mathbf{P}_{1}\right) \\
\phi_{2}(t)=\phi=\phi(t) & \left(\text { Phase of } \mathbf{P}_{2}\right) \\
\phi_{0}(t)=\phi_{1}(t)-\phi_{2}(t)=\phi_{0} & \left(\text { Phase difference of } \mathbf{P}_{1} \text { and } \mathbf{P}_{2}\right)
\end{array}
$$

At each phase $\phi$ (2), the circles have vector radii $\vec{r}_{1}$ (4) - (5) and $\overrightarrow{\boldsymbol{r}}_{2}(6)$, with constant magnitudes $r_{1}(7)$ and $r_{2}(7)$, respectively, and centers of these two circles are displaced by a constant or variable vector $\vec{\ell}(\phi)(8)$ with magnitude $\ell(\phi)(9)$, which is also the scalar distance between centers of the two circles.

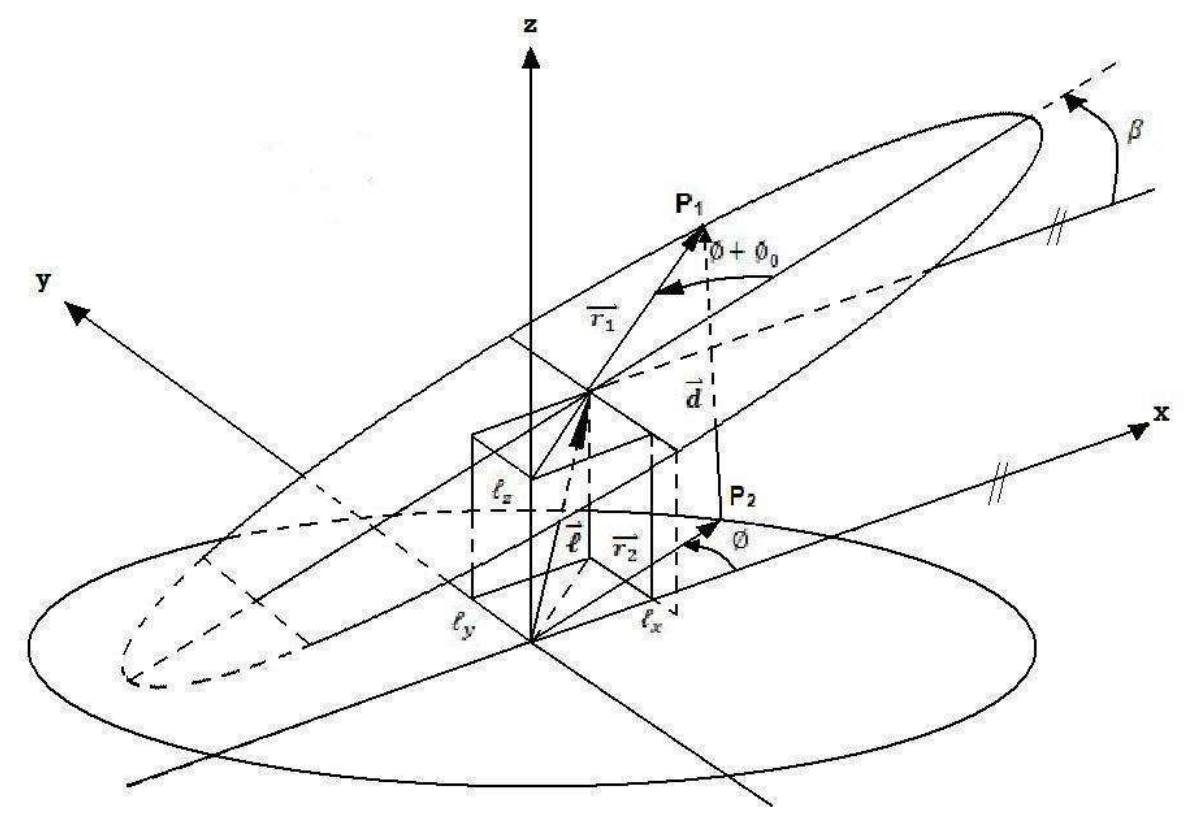

Figure 1 Distance Between Points $\mathbf{P}_{1}$ and $\mathbf{P}_{2}$ Around Two Different Circles in Space 
Note that in all our subsequent operations, we take the "square of a vector" as the "dot product of the vector with itself", which amounts to the scalar "square of the magnitude" for any vector.

$$
\begin{gathered}
\overrightarrow{\boldsymbol{r}}_{1}=\overrightarrow{\boldsymbol{r}}_{1}\left(\phi+\phi_{0}\right)=\hat{\boldsymbol{x}} r_{1} \operatorname{Cos}\left(\phi+\phi_{0}\right) \operatorname{Cos} \beta+\hat{\boldsymbol{y}} r_{1} \operatorname{Sin}\left(\phi+\phi_{0}\right)+\hat{\boldsymbol{z}} r_{1} \operatorname{Cos}\left(\phi+\phi_{0}\right) \operatorname{Sin} \beta \\
\overrightarrow{\boldsymbol{r}}_{1}=\overrightarrow{\boldsymbol{r}}_{1}[\phi(t)]=\left(\hat{\boldsymbol{x}} r_{1} \operatorname{Cos} \beta \operatorname{Cos}\left[\phi_{0}(t)\right]+\hat{\boldsymbol{y}} r_{1} \operatorname{Sin}\left[\phi_{0}(t)\right]+\hat{\boldsymbol{z}} r_{1} \operatorname{Sin} \beta \operatorname{Cos}\left[\phi_{0}(t)\right]\right) \operatorname{Cos}[\phi(t)]+ \\
\left(-\hat{\boldsymbol{x}} r_{1} \operatorname{Cos} \beta \operatorname{Sin}\left[\phi_{0}(t)\right]+\hat{\boldsymbol{y}} r_{1} \operatorname{Cos}\left[\phi_{0}(t)\right]-\hat{\boldsymbol{z}} r_{1} \operatorname{Sin} \beta \operatorname{Sin}\left[\phi_{0}(t)\right]\right) \operatorname{Sin}[\phi(t)] \\
\overrightarrow{\boldsymbol{r}}_{2}=\overrightarrow{\boldsymbol{r}}_{2}[\phi(t)]=\hat{\boldsymbol{x}} r_{2} \operatorname{Cos}[\phi(t)]+\hat{\boldsymbol{y}} r_{2} \operatorname{Sin}[\phi(t)] \\
r_{1}=\left|\overrightarrow{\boldsymbol{r}}_{1}\right|=\sqrt{\overrightarrow{\boldsymbol{r}}_{1} \cdot \overrightarrow{\boldsymbol{r}}_{1}}=\sqrt{r_{1}^{2}} \quad ; \quad r_{2}=\left|\overrightarrow{\boldsymbol{r}}_{2}\right|=\sqrt{\overrightarrow{\boldsymbol{r}}_{2} \cdot \overrightarrow{\boldsymbol{r}}_{2}}=\sqrt{r_{2}^{2}} \\
\vec{\ell}=\vec{\ell}[\phi(t)]=\hat{\boldsymbol{x}} \ell_{x}[\phi(t)]+\hat{\boldsymbol{y}} \ell_{y}[\phi(t)]+\hat{\boldsymbol{z}} \ell_{z}[\phi(t)] \\
|\vec{\ell}(\phi)|=\ell(\phi)=\sqrt{\ell^{2}(\phi)}=\sqrt{\hat{\ell}(\phi) \cdot \vec{\ell}(\phi)} \quad ; \quad \ell^{2}(\phi)=\vec{\ell}(\phi) \cdot \vec{\ell}(\phi)=\ell_{x}^{2}(\phi)+\ell_{y}^{2}(\phi)+\ell_{z}^{2}(\phi)
\end{gathered}
$$

The vector distance from any point $\mathbf{P}_{2}$ to $\mathbf{P}_{1}$ is expressed as $\overrightarrow{\boldsymbol{d}}(\phi)$ (10) - (12), where its magnitude $d(\phi)(13)$ is also the scalar distance between points $\mathbf{P}_{1}$ and $\mathbf{P}_{2}$ on the two respective circles.

$$
\begin{aligned}
& \overrightarrow{\boldsymbol{d}}(\phi)= \overrightarrow{\boldsymbol{r}_{1}}\left(\phi+\phi_{0}\right)-\overrightarrow{\boldsymbol{r}_{2}}(\phi)+\vec{\ell}(\phi)=\overrightarrow{\boldsymbol{r}_{1}}-\overrightarrow{\boldsymbol{r}_{2}}+\vec{\ell}=\overrightarrow{\boldsymbol{a}} \operatorname{Cos} \phi+\overrightarrow{\boldsymbol{b}} \operatorname{Sin} \phi+\vec{\ell}(\phi)=\overrightarrow{\mathbf{X}}(\phi)+\overrightarrow{\mathbf{Y}}(\phi)+\vec{\ell}(\phi) \\
& \overrightarrow{\boldsymbol{d}}(\phi)= \hat{\boldsymbol{x}}\left[r_{1} \operatorname{Cos}\left(\phi+\phi_{0}\right) \operatorname{Cos} \beta-r_{2} \operatorname{Cos} \phi+\ell_{x}(\phi)\right]+\hat{\boldsymbol{y}}\left[r_{1} \operatorname{Sin}\left(\phi+\phi_{0}\right)-r_{2} \operatorname{Sin} \phi+\ell_{y}(\phi)\right] \\
&+\hat{\boldsymbol{z}}\left[r_{1} \operatorname{Cos}\left(\phi+\phi_{0}\right) \operatorname{Sin} \beta+\ell_{z}(\phi)\right] \\
& \overrightarrow{\boldsymbol{d}}[\phi(t)]= {\left[\hat{\boldsymbol{x}}\left(r_{1} \operatorname{Cos} \beta \operatorname{Cos}\left[\phi_{0}(t)\right]-r_{2}\right)+\hat{\boldsymbol{y}} r_{1} \operatorname{Sin}\left[\phi_{0}(t)\right]+\hat{\boldsymbol{z}} r_{1} \operatorname{Sin} \beta \operatorname{Cos}\left[\phi_{0}(t)\right]\right] \operatorname{Cos}[\phi(t)]+} \\
& {\left[-\hat{\boldsymbol{x}} r_{1} \operatorname{Cos} \beta \operatorname{Sin}\left[\phi_{0}(t)\right]+\hat{\boldsymbol{y}}\left(r_{1} \operatorname{Cos}\left[\phi_{0}(t)\right]-r_{2}\right)-\hat{\boldsymbol{z}} r_{1} \operatorname{Sin} \beta \operatorname{Sin}\left[\phi_{0}(t)\right]\right] \operatorname{Sin}[\phi(t)]+} \\
& {\left[\hat{\boldsymbol{x}} \ell_{x}[\phi(t)]+\hat{\boldsymbol{y}} \ell_{y}[\phi(t)]+\hat{\boldsymbol{z}} \ell_{z}[\phi(t)]\right] } \\
& d(\phi)=|\overrightarrow{\boldsymbol{d}}(\phi)|=\sqrt{d^{2}(\phi)}=\sqrt{\overrightarrow{\boldsymbol{d}}(\phi) \cdot \overrightarrow{\boldsymbol{d}}(\phi)}
\end{aligned}
$$

Vector distance $\overrightarrow{\boldsymbol{d}}(\phi)$ (12) at any phase $\phi$ (2) can equivalently be expressed as in $\overrightarrow{\boldsymbol{d}}(\phi)$ (10) in terms of virtual vectors $\overrightarrow{\mathbf{X}}(\phi)$ (14) and $\overrightarrow{\mathbf{Y}}(\phi)$ (15) that we have defined, with magnitudes are $X(\phi)$ (14) and $Y(\phi)$ (15), respectively, as well as vectors $\overrightarrow{\boldsymbol{a}}$ (16) and $\overrightarrow{\boldsymbol{b}}$ (17) we have defined, with magnitudes $a$ (18) 
and $b$ (19), respectively. When the phase difference $\phi_{0}$ (3) is constant for all $\phi(2), a(18)$ and $b$ (19) are also constant, but if $\left[\phi_{0}=\phi_{0}(t)\right](3)$ is time $(t)$ dependent, $a(t)(18)$ and $b(t)(19)$ are as well.

$$
\begin{array}{r}
\overrightarrow{\mathbf{X}}(\phi)=\overrightarrow{\boldsymbol{a}} \operatorname{Cos} \phi \quad ; \quad \overrightarrow{\mathbf{X}}(\phi) \cdot \overrightarrow{\mathbf{X}}(\phi)=X^{2}(\phi)=\overrightarrow{\boldsymbol{a}} \cdot \overrightarrow{\boldsymbol{a}} \operatorname{Cos}^{2} \phi=a^{2} \operatorname{Cos}^{2} \phi \quad ;|\overrightarrow{\mathbf{X}}(\phi)|=X(\phi) \\
\overrightarrow{\mathbf{Y}}(\phi)=\overrightarrow{\boldsymbol{b}} \operatorname{Sin} \phi \quad ; \quad \overrightarrow{\mathbf{Y}}(\phi) \cdot \overrightarrow{\mathbf{Y}}(\phi)=Y^{2}(\phi)=\overrightarrow{\boldsymbol{b}} \cdot \overrightarrow{\boldsymbol{b}} \operatorname{Sin}^{2} \phi=b^{2} \operatorname{Sin}^{2} \phi \quad ;|\overrightarrow{\mathbf{Y}}(\phi)|=Y(\phi) \\
\overrightarrow{\boldsymbol{a}}=\overrightarrow{\boldsymbol{a}}(t)=\hat{\boldsymbol{x}}\left\{r_{1} \operatorname{Cos} \beta \operatorname{Cos}\left[\phi_{0}(t)\right]-r_{2}\right\}+\hat{\boldsymbol{y}} r_{1} \operatorname{Sin}\left[\phi_{0}(t)\right]+\hat{\boldsymbol{z}} r_{1} \operatorname{Sin} \beta \operatorname{Cos}\left[\phi_{0}(t)\right]=\left(\overrightarrow{\boldsymbol{r}}_{1}-\overrightarrow{\boldsymbol{r}_{2}}\right)(\phi=0) \\
\overrightarrow{\boldsymbol{b}}=\overrightarrow{\boldsymbol{b}}(t)=-\hat{\boldsymbol{x}} r_{1} \operatorname{Cos} \beta \operatorname{Sin}\left[\phi_{0}(t)\right]+\hat{\boldsymbol{y}}\left\{r_{1} \operatorname{Cos}\left[\phi_{0}(t)\right]-r_{2}\right\}-\hat{\boldsymbol{z}} r_{1} \operatorname{Sin} \beta \operatorname{Sin}\left[\phi_{0}(t)\right]=\left(\overrightarrow{\boldsymbol{r}_{1}}-\overrightarrow{\boldsymbol{r}_{2}}\right)\left(\phi=\frac{\pi}{2}\right) \\
\overrightarrow{\boldsymbol{a}}(t) \cdot \overrightarrow{\boldsymbol{a}}(t)=a^{2}(t)=r_{1}^{2}-2 r_{1} r_{2} \operatorname{Cos} \beta \operatorname{Cos}\left[\phi_{0}(t)\right]+r_{2}^{2} \quad ; \quad a=a(t)=|\overrightarrow{\boldsymbol{a}}(t)|=\sqrt{\overrightarrow{\boldsymbol{a}}(t) \cdot \overrightarrow{\boldsymbol{a}}(t)} \\
\overrightarrow{\boldsymbol{b}}(t) \cdot \overrightarrow{\boldsymbol{b}}(t)=b^{2}(t)=r_{1}^{2}-2 r_{1} r_{2} \operatorname{Cos}\left[\phi_{0}(t)\right]+r_{2}^{2} \quad ; \quad b=b(t)=|\overrightarrow{\boldsymbol{b}}(t)|=\sqrt{\overrightarrow{\boldsymbol{b}}(t) \cdot \overrightarrow{\boldsymbol{b}}(t)}(19)
\end{array}
$$

According to the definitions of vectors $\overrightarrow{\mathbf{X}}(\phi)$ (14), $\overrightarrow{\mathbf{Y}}(\phi)$ (15), $\overrightarrow{\boldsymbol{a}}$ (16), and $\overrightarrow{\boldsymbol{b}}$ (17), the relation in (20) is valid and holds for all $\phi$ (2) due to the given trigonometric identity in Eq. (20). Therefore, the relation in Eq. (20) reveals the validity of Eq. (22) and Eq. (21) for vector pair $[\overrightarrow{\mathbf{X}}(\phi), \overrightarrow{\mathbf{Y}}(\phi)]$ (14) - (15) and its magnitude pair $[X(\phi), Y(\phi)](14)$ - (15), respectively.

$$
\begin{gathered}
\frac{\overrightarrow{\mathbf{X}}(\phi) \cdot \overrightarrow{\mathbf{X}}(\phi)}{\overrightarrow{\boldsymbol{a}} \cdot \overrightarrow{\boldsymbol{a}}}+\frac{\overrightarrow{\mathbf{Y}}(\phi) \cdot \overrightarrow{\mathbf{Y}}(\phi)}{\overrightarrow{\boldsymbol{b}} \cdot \overrightarrow{\boldsymbol{b}}}=\frac{X^{2}(\phi)}{a^{2}}+\frac{Y^{2}(\phi)}{b^{2}}=\operatorname{Cos}^{2} \phi+\operatorname{Sin}^{2} \phi=1 \\
\frac{X^{2}(\phi)}{a^{2}}+\frac{Y^{2}(\phi)}{b^{2}}=1 \quad \text { (Definition of Scalar Ellipse in 2-Dimensions) }
\end{gathered}
$$

$$
\frac{\overrightarrow{\mathbf{X}}(\phi) \cdot \overrightarrow{\mathbf{X}}(\phi)}{\overrightarrow{\boldsymbol{a}} \cdot \overrightarrow{\boldsymbol{a}}}+\frac{\overrightarrow{\mathbf{Y}}(\phi) \cdot \overrightarrow{\mathbf{Y}}(\phi)}{\overrightarrow{\boldsymbol{b}} \cdot \overrightarrow{\boldsymbol{b}}}=1
$$

As Eq. (21) is the defining equation of an ellipse in two dimensions, where $a(18)$ is the semi major axis and $b$ (19) is the semi minor axis of the ellipse when $(a>b)$ (23), and vice versa, with Eq. (21) reducing to the special case of a circle equation when $(a=b)$ (23), we claim Eq. (22) indicates that the vector pair $[\overrightarrow{\mathbf{X}}(\phi), \overrightarrow{\mathbf{Y}}(\phi)](14)$ - (15) defines points on a vector ellipse in three dimensions in the most 
general case, with $\overrightarrow{\boldsymbol{a}}$ (16) and $\overrightarrow{\boldsymbol{b}}$ (17) as its semi major axis semi minor axis vectors. In other words, vector distance $\overrightarrow{\boldsymbol{d}}(\phi)$ (12) between two points $\mathbf{P}_{1}$ and $\mathbf{P}_{2}$ moving around two circles, whose centers are displaced by a constant or variable vector $\vec{\ell}(\phi)$ (8), can equivalently be mathematically expressed and interpreted as the distance $\overrightarrow{\boldsymbol{d}}(\phi)$ (10) of points on a virtual vector ellipse, whose locations with respect to a virtual origin at each $\phi$ (2) are determined by the sum of vector pair $[\overrightarrow{\mathbf{X}}(\phi), \overrightarrow{\mathbf{Y}}(\phi)]$ (14) - (15), from another point displaced from the same virtual origin of the ellipse by a constant or variable vector $[-\vec{\ell}(\phi)]$ (8), where $\overrightarrow{\boldsymbol{a}}$ (16) and $\overrightarrow{\boldsymbol{b}}$ (17) are the fixed or variable semi major axis semi minor axis vectors of the vector ellipse. This result is mathematically valid even when the phase difference $\left[\phi_{0}=\phi_{0}(t)\right]$ (3) is a variable function of time $(t)$. This revelation ${ }^{1,2}$ is the core and most significant finding of our research. Moreover, we have also mathematically introduced the concept of a "vector ellipse" in Eq. (22). More detail on our research results can be found in our book ${ }^{1}$.

$$
\left\{\begin{array}{l}
a(t)>b(t) \Rightarrow \overrightarrow{\boldsymbol{a}}(t) \text { is semi-major axis \& } \overrightarrow{\boldsymbol{b}}(t) \text { is semi-minor axis of vector ellipse } \\
a(t)<b(t) \Rightarrow \overrightarrow{\boldsymbol{b}}(t) \text { is semi-major axis \& } \overrightarrow{\boldsymbol{a}}(t) \text { is semi-minor axis of vector ellipse } \\
a(t)=b(t) \Rightarrow \overrightarrow{\boldsymbol{a}}(t) \text { and } \overrightarrow{\boldsymbol{b}}(t) \text { are radii of vector circle }
\end{array}\right.
$$

Instantaneous focal distance $c$ (24) of the vector ellipse can be determined using Eq.s (18) - (19), and instantaneous eccentricity $e$ (25) of the vector ellipse can be found using Eq.s (18) - (19) and (24).

$$
\begin{aligned}
& c=c(t)=\sqrt{c^{2}(t)}=\sqrt{\left|a^{2}(t)-b^{2}(t)\right|}=\sqrt{2 r_{1} r_{2}(1-\operatorname{Cos} \beta) \mid \operatorname{Cos}\left[\phi_{0}(t)\right]} \quad \text { (Focal Distance) } \\
& e=e(t)=\left\{\begin{array}{l}
\frac{c(t)}{a(t)}=\sqrt{\frac{2 r_{1} r_{2}(1-\operatorname{Cos} \beta)\left|\operatorname{Cos}\left[\phi_{0}(t)\right]\right|}{r_{1}^{2}-2 r_{1} r_{2} \operatorname{Cos} \beta \operatorname{Cos}\left[\phi_{0}(t)\right]+r_{2}^{2}}} \quad \text { if } \quad a(t)>b(t) \\
\frac{c(t)}{b(t)}=\sqrt{\frac{2 r_{1} r_{2}(1-\operatorname{Cos} \beta) \mid \operatorname{Cos}\left[\phi_{0}(t)\right]}{r_{1}^{2}-2 r_{1} r_{2} \operatorname{Cos}\left[\phi_{0}(t)\right]+r_{2}^{2}}} \quad \text { if } \quad a(t)<b(t)
\end{array}\right.
\end{aligned}
$$

The major consequence in physics, of this analysis and mathematical results in Eq.s (1) to (25) for moving points $\mathbf{P}_{1}$ and $\mathbf{P}_{2}$ on the two respective circles, is that "Particles or bodies moving around different circular orbits in space see themselves positioned on an elliptical route with respect to each other, these elliptical orbits having fixed or time varying semi major and semi minor axes depending on the time dependencies of the angular velocities of the particles or bodies, where the particles or bodies 
instantaneously observe their counterpart virtually positioned at a fixed point in space, whose position is determined by the distance vector between centers of their individual circular orbits around which they revolve."

This mathematical revelation has very far-reaching consequences in physics. It would lead to the formulation of a fundamental new model of motion for moving bodies in the Universe, including the Sun, the Planets, and the Satellites in the Solar System and elsewhere, as well as at particle and subatomic level, where "all bodies move at some angular velocity around their own circular orbits of revolution with different radii and centers of revolution in space", but "each appear to be moving as if in elliptical orbits with respect to each other, where they see the other body located at a fixed or variable point in their virtual mutual plane of motion". This subsequently would lead to more insight into forces in nature.

Based on the demonstrated mathematical analysis, as they exhibit distinct elliptic orbits relative to one another over time, we make the assertion that Sun, Earth, and Moon must each be revolving in their individual circular orbits of revolution in space. With this expectation, orbital parameters of Sun, Earth, and Moon are calculated ${ }^{1,3}$ based on observed distance data between Sun and Earth, and Earth and Moon, and also using analytical methods developed as part of this research to an approximation, presented in Eq.s (26) to (57). Our analysis revealed additional results aligned with observation and thus supporting our assertion that Sun, Earth, and Moon must actually be revolving in individual circular orbits, and these can be found in our book ${ }^{1}$.

Based on observations and our findings as a result of our research, we have discovered and make the broader assertion that "Moving bodies in space must be revolving around individual circular orbits within their revolution plane, which determine their individual equatorial planes. The normal to its revolution plane and the direction of motion of the body around its individual circular orbit determine the axis of self revolution according to 'right hand rule'. The direction of self rotation axis of each body in space is also determined according to 'right hand rule' based on the direction of self rotation of the body. Self revolution axis and self rotation axis of each moving body in space must be aligned along the normal of its equatorial plane. As all moving bodies in space must obey 'Faraday's Law of Induction', we assert that all bodies in space possibly behave like macro scale charged particles moving in external magnetic fields with changing flux through the area of their individual circular orbit of revolution while moving in clockwise direction (Revolution Orbit 'West' to Revolution Orbit 'East') from a vantage point above their North Pole, which we expect must be the main factor that triggers their self rotation in the reverse (counterclockwise) direction (Self Rotation 'West' to Self Rotation 'East') as seen from a vantage point above their North Pole, hence the bodies in the Universe have two Easts and two Wests in this context, where we always accept the North Pole of a body or a revolution to be the pole rotating in 
counterclockwise direction according to 'right hand rule'. Based on this assertion, we also infer that the axial tilt of a body in its motion relative to another body must topologically be based on the orbital inclination angle between their planes of individual circular orbits of revolution in space." This assertion is also supported by our research results for the Sun-Earth-Moon system, details of which can be found in our book ${ }^{1}$.

We have found ${ }^{1,3}$ out that the actual Sun-Earth-Moon topology in space can be represented roughly as in the configuration of Figure 2, not to scale, which in fact is the Sun-Earth configuration of Figure 1 superimposed on the Earth-Moon configuration of Figure 1. Note that in the configuration of Figure 2 with Cartesian $(\hat{\boldsymbol{x}}, \hat{\boldsymbol{y}}, \hat{\boldsymbol{z}})$ coordinates, the plane of Earth's individual circular orbit of revolution is taken to be horizontal $\hat{\boldsymbol{x}}-\hat{\boldsymbol{y}}$ plane, and the direction of the individual circular orbit of revolution of Earth is taken to be counterclockwise in $[+\phi(t)]$ (2) direction looking down from $\hat{\boldsymbol{z}}$-axis onto $\hat{\boldsymbol{x}}-\hat{\boldsymbol{y}}$ plane, and this corresponds to clockwise direction from a vantage point above the North Pole of the Earth, as our calculations have revealed that $\left(\hat{\boldsymbol{u}}_{\text {Earth } \perp}=-\hat{\boldsymbol{z}}\right)(26)$ is the unit vector in the direction of self rotation axis of Earth in this Figure 2 configuration, which is also the direction of the North Pole of Earth.

$$
\hat{\boldsymbol{u}}_{\text {Earth } \perp}=-\hat{\boldsymbol{z}} \quad(\text { North Pole unit vector of Earth })
$$

The inclination angle of the plane of individual circular orbit of revolution of the Sun with respect to the plane of individual circular orbit revolution of the Earth is found as $\beta_{\text {Sun-Earth }}$ (27).

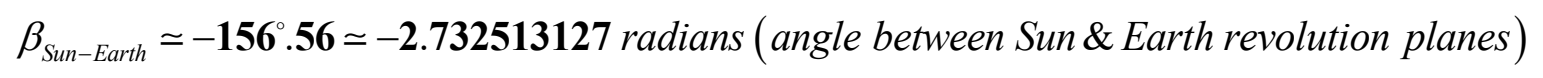

Our analysis based on observation has also led to the conclusion that the pole vector of the Sun that is angle-wise closer to the Earth's North Pole should be the Sun's South Pole vector. Subsequently, we have found the direction of the Sun's North Pole, also the unit vector in the direction of the self rotation axis of the Sun, to be $\left(\hat{\boldsymbol{u}}_{\operatorname{Sun} \perp}=-\hat{\boldsymbol{x}} \operatorname{Sin} \beta_{\text {Sun-Earth }}+\hat{\boldsymbol{z}} \operatorname{Cos} \beta_{\text {Sun-Earth }}\right)$ (28) in the configuration of Figure 2. Furthermore, Sun's tilt with respect to the distance vector $[-\overrightarrow{\boldsymbol{d}}(\phi)](10)$ from Sun to Earth is around $90^{\circ}$ throughout a year according to our calculations, and we have also calculated the Sun's wobble angle and sideways swing angle as observed from Earth throughout a year in more detail in our book ${ }^{1}$.

$$
\hat{\boldsymbol{u}}_{\text {Sun } \perp}=-\hat{\boldsymbol{x}} \operatorname{Sin} \beta_{\text {Sun-Earth }}+\hat{\boldsymbol{z}} \operatorname{Cos} \beta_{\text {Sun-Earth }} \quad \text { (North Pole unit vector of Sun) }
$$


Our analysis has revealed that inclination angle of the plane of individual circular orbit of revolution of the Moon with respect to the plane of individual circular orbit revolution of Earth is $\beta_{\text {Moon-Earth }}$ whereas the direction of self rotation axis of the Moon, as is also the direction of North Pole of the Moon in the configuration of Figure 2, is $\left(\hat{\boldsymbol{u}}_{\text {Moon }}=\hat{\boldsymbol{x}} \operatorname{Sin} \beta_{\text {Moon-Earth }}-\hat{\boldsymbol{z}} \operatorname{Cos} \beta_{\text {Moon-Earth }}\right)$ (30).

$$
\begin{aligned}
& \beta_{\text {Moon-Earth }} \simeq 24^{\circ} \quad(\text { angle between Moon \& Earth revolution planes }) \\
& \hat{\boldsymbol{u}}_{\text {Moon } \perp}=\hat{\boldsymbol{x}} \operatorname{Sin} \beta_{\text {Moon-Earth }}-\hat{\boldsymbol{z}} \operatorname{Cos} \beta_{\text {Moon-Earth }} \quad(\text { North Pole unit vector of Moon })
\end{aligned}
$$

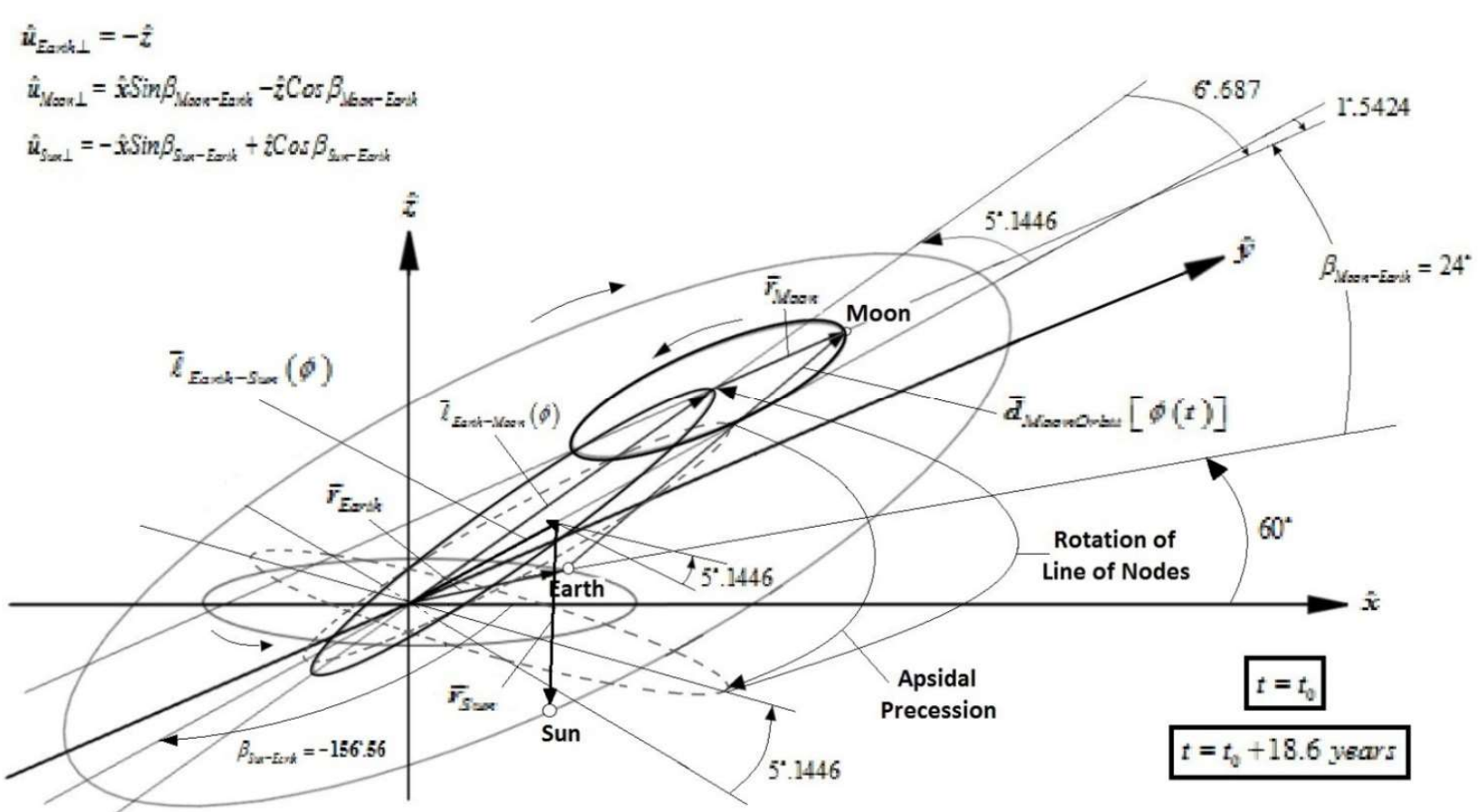

Figure 2 Sun-Earth-Moon Configuration based on Circular Orbit Model

We have found that the constant phase difference is $\left(\phi_{0, \text { Earth-Sun }} \simeq \mathbf{- 1 5 1 ^ { \circ } . 1 1 )}\right.$ (31) between Sun and Earth in their individual orbits of revolution throughout a year, such that in its own cycle of individual revolution, Earth is moving ahead of the Sun in its own cycle of individual revolution by a phase difference of $151^{\circ} .11$ (31). In the Sun-Earth-Moon configuration of Figure 2, we have discovered that the phase $\left[\phi(t)=\phi_{\text {Earth-Sun }}\right]$ (32) of Earth in its individual circular orbit of revolution in its motion relative to the Sun in the Sun-Earth configuration of Figure 1 at any date is about $60^{\circ}$ ahead of the phase 
$\left[\phi(t)=\phi_{\text {Earth-Moon }}\right]$ (32) of Earth in its individual circular orbit of revolution in its motion relative to the Moon in the Earth-Moon configuration of Figure 1.

$$
\begin{array}{cl}
\phi_{0, \text { Earth-Sun }}=-\mathbf{2 . 6 3 7 3 6 9 9 7 1 9 8 9 5 1} \mathrm{rad}=-\mathbf{1 5 1} .11 & \text { (Phase difference for Sun-Earth system }) \\
\left.\phi_{\text {Earth-Sun }} \simeq \phi_{\text {Earth-Moon }}+60^{\circ} \quad \text { (at any date of the year }\right)
\end{array}
$$

We have obtained the following radii magnitudes $r_{\text {Sun }}$ (33), $r_{\text {Earth }}$ (34), and $r_{\text {Moon }}$ (35) for individual circular orbits of revolution of the Sun, the Earth, and the Moon, respectively, in terms of kilometers $(\mathrm{km})$ and astronomical units (au), based on observed yearly Earth to Sun distance data as well as Earth to Moon distance data, and using an analytical method we have developed to calculate orbital parameters of the heavenly bodies, to some approximations. Even if we have obtained approximate values with the analytical calculation, the result we have reached is important in comparing the order of magnitude of the radii of individual circular orbits of revolution of Sun, Earth, and Moon, as $\left(r_{\text {Earth }} \ll r_{\text {Moon }} \ll r_{\text {Sun }}\right)$ (36).

$$
\begin{aligned}
& r_{\text {Sun }}=\mathbf{1 . 0 0 0 0 7 8 6 9 1 2 8 4 4 6} \text { au }=149,609,642.749 \mathrm{~km} \quad \text { (radius of Sun's revolution orbit) } \\
& r_{\text {Earth }}=\mathbf{0 . 0 0 0 0 8 2 6 9 1 5 3 4 0 9 5 0 7 2 1} a u=\mathbf{1 2 , 3 7 0 . 4 7 7 ~} \mathrm{km} \text { (radius of Earth's revolution orbit) } \\
& r_{\text {Moon }} \simeq \mathbf{3 , 3 0 2 , 0 0 9 . 7 8 1} \mathrm{km} \simeq \mathbf{0 . 0 2 2 0 7 2 5 7 2 0 6 0 4 1 6 1} \text { au (radius of Moon's revolution orbit) } \\
& \left(r_{\text {Eart }} \simeq 12,370.477 \mathrm{~km}\right) \ll\left(r_{\text {Moon }} \simeq 3,302,009.781 \mathrm{~km}\right) \ll\left(r_{\text {Sun }} \simeq 149,609,642.749 \mathrm{~km}\right)
\end{aligned}
$$

The most significant and interesting implication based on the orbital parameter values found for the Sun, the Earth, and the Moon is, the obtained results indicate that the Earth is not revolving around the Sun, and the Moon is not directly revolving around the Earth, but the results in fact show that the Sun is revolving around a larger individual circular orbit which encompasses the individual circular orbits of the Earth and the Moon with an orbital inclination, where the centers of revolution of each are shifted with respect to each other's. The center of revolution of the Moon is in continuous harmonic motion relative to centers of revolution of other bodies, mainly over a cycle of approximately 18.6 years with respect to the center of Earth's revolution, which is analyzed in detail in our book ${ }^{1}$. Hence, we have concluded that planets do not necessarily revolve around stars, and Earth is not revolving around the Sun. These findings raise the need to reevaluate existing theories about formation, expected motional behavior, and topologies of solar systems observed in the Universe in general as well as our Solar System, bringing new vision. 
Phase values $\left[\phi(t)=\phi_{\text {Earth-Sun }}\right]$ (2) of Earth for equinoxes, solstices, and dates of minimum and maximum Earth to Sun distances, as well as dates of some significant phases in the Earth-Sun yearly cycle are listed in Eq.s (37) to (46), in the Sun-Earth configuration of Figure 1.

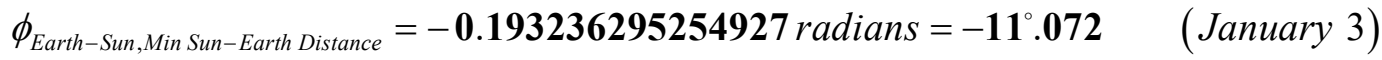

$$
\begin{aligned}
& \phi_{\text {Earth-Sun }}=\mathbf{0 . 0 0 0 5 8 6 7 3 2 5 4 9 4 9 7 2 6} \text { radians }=\mathbf{0}^{\circ} .034 \simeq 0 \\
& \phi_{\text {Earth-Sun,Spring Equinox }}=\mathbf{1 . 0 2 6 7 6 7 7 1 6 7 1 5 7 4} \text { radians }=\mathbf{5 8}^{\circ} .829
\end{aligned}
$$

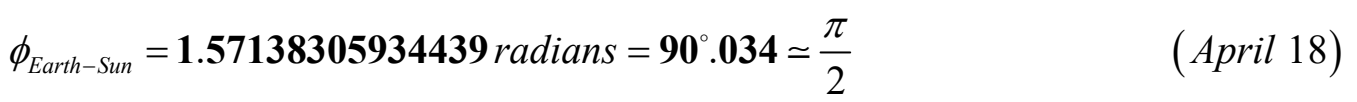

$$
\begin{aligned}
& \phi_{\text {Earth-Sun,Summer Solstice }}=-\phi_{0, \text { Earth-Sun }}=\mathbf{2 . 6 3 7 3 6 9 9 7 1 9 8 9 5 1} \mathrm{rad}=\mathbf{1 5 1}^{\circ} . \mathbf{1 1} \quad \text { (June 21) }
\end{aligned}
$$

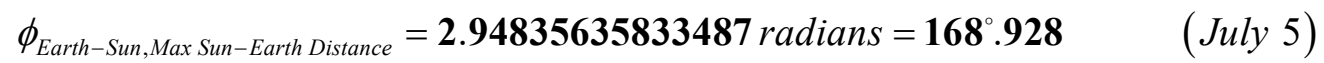

$$
\begin{aligned}
& \phi_{\text {Earth-Sun }}=\mathbf{3 . 1 4 2 1 7 9 3 8 6 1 3 9 2 9} \text { radians }=180^{\circ} .034 \simeq \pi \\
& \phi_{\text {Earth-Sun }, \text { Autumn Equinox }}=-\mathbf{2 . 0 5 4 8 8 1 7 6 7 0 8 2 2 9} \text { radians }=\mathbf{2 4 2}^{\circ} . \mathbf{2 6 4}=-\mathbf{1 1 7}^{\circ} . \mathbf{7 3 6} \quad(\text { September } 22)
\end{aligned}
$$

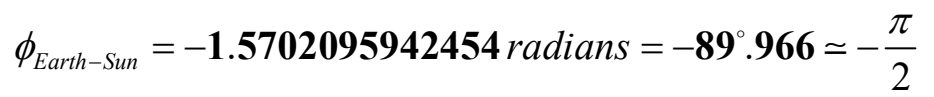

$$
\begin{aligned}
& \text { (January 17) (38) } \\
& \text { (March 20) } \\
& \text { (July 18) }
\end{aligned}
$$

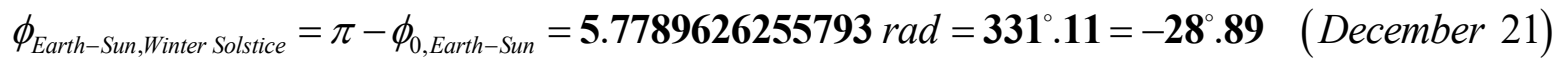

For the relative motion ellipse of Sun-Earth system, we have also determined the fixed semi minor axis and semi major axis vectors $\left[\overrightarrow{\boldsymbol{a}}(t)=\overrightarrow{\boldsymbol{a}}_{\text {Earth-Sun }}\right]$ (47) and $\left[\overrightarrow{\boldsymbol{b}}(t)=\overrightarrow{\boldsymbol{b}}_{\text {Earth-Sun }}\right]$ (48), respectively, as well as values of their fixed magnitudes $\left[a(t)=a_{\text {Earth-Sun }}\right]$ (49) and $\left[b(t)=b_{\text {Earth-Sun }}\right]$ (50), respectively, with their focal distance $\left[c(t)=c_{\text {Earth-Sun }}\right]$ (51) and eccentricity $\left[e(t)=e_{\text {Earth-Sun }}\right]$ (52), in the Sun-Earth configuration of Figure 1.

$$
\begin{gathered}
\overrightarrow{\boldsymbol{a}}_{\text {Earth-Sun }}=\hat{\boldsymbol{x}} 120,168,467.040 \mathrm{~km}-\hat{\boldsymbol{y}} 72,280,843.623 \mathrm{~km}+\hat{\boldsymbol{z}} 52,106,536.351 \mathrm{~km} \\
\overrightarrow{\boldsymbol{b}}_{\text {Earth-Sun }}=-\hat{\boldsymbol{x}} 66,316,021.722 \mathrm{~km}-\hat{\boldsymbol{y}} 131,002,922.948 \mathrm{~km}-\hat{\boldsymbol{z}} 28,752,488.898 \mathrm{~km}
\end{gathered}
$$




$$
\begin{aligned}
& a_{E a r t h-S u n}=1.00001226586661 \text { au }=149,599,705.647527 \mathrm{~km} \\
& \text { (Sun-Earth semi minor axis magnitude) } \\
& b_{\text {Earth-Sun }}=1.00015109267839 \mathrm{au}=\mathbf{1 4 9}, \mathbf{6 2 0}, \mathbf{4 7 3 . 8 4 2 9 6 5 \mathrm { km }} \\
& \text { (Sun-Earth semi major axis magnitude) }
\end{aligned}
$$

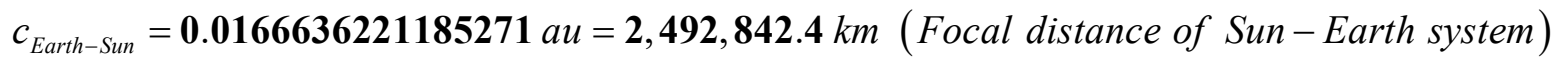

$$
\begin{aligned}
& e_{E a r t h-S u n}=\frac{c_{E a r h-S u n}}{b_{E a r h-S u n}}=\mathbf{0 . 0 1 6 6 6 1 1 0 4 7 4 7 5 8 5 8} \quad(\text { Eccentricity of Sun }- \text { Earth system })
\end{aligned}
$$

We have discovered that the magnitude $\left[\ell(\phi)=\ell_{\text {Earth-Sun }}(\phi)\right]$ (9) of the vector distance $\left\{\vec{\ell}[\phi(t)]=\vec{\ell}_{\text {Earth-Sun }}(\phi)\right\}(8)$ between the centers of individual circular orbits of revolutions of Earth and Sun exhibits three distinct main oscillation frequencies over a yearly Sun-Earth cycle. One oscillation is about 12 times a year, which is apparently based on the impact of the relative Earth-Moon motion. The second oscillation is seemingly based on the daily self rotation of Earth around its own axis. The possible cause of the third oscillation, which is about 4 times over a Sun-Earth year, may be the impact of relative Sun-Mercury motion which has about four cycles over a Sun-Earth year. It is possible that the Sun and Mercury form a twin or binary system of bodies in space, in which Mercury behaves as the Sun's satellite, similar to Earth-Moon system, and this also implies the possibility that the individual circular orbits of revolution of the Sun and Mercury may be revolving around each other, to be confirmed when Mercury orbital parameters are determined. Our analysis also implies it is possible that relative motions of other planets and their satellites, and even other moving bodies in the Solar System, may impact the relative motion of Sun and Earth in terms of additional minor oscillations of $\left[\ell(\phi)=\ell_{\text {Earth-Sun }}(\phi)\right]$ (9), which can be observed only over longer periods than a Sun-Earth year.

Maximum distance $\left[\ell_{\text {Earth-Sun }}(\phi)\right]_{\text {Max }}(53)$ between centers of individual circular orbits of revolution of the Sun and the Earth occur on February 23, May 26, August 25, and November 24, and minimum distance $\left[\ell_{\text {Earth-Sun }}(\phi)\right]_{\text {Min }}(54)$ between centers of individual circular orbits of revolution of the Sun and the Earth occur on January 1, April 2, July 2, and October 1, over a yearly Sun-Earth cycle.

$$
\begin{aligned}
& \left.\left[\ell_{\text {Earth-Sun }}(\phi)\right]_{\text {Max }}=\mathbf{0 . 0 1 6 8 3 4 6 1 8 4 9 5 4 4 4 6} \text { au (23 February, } 26 \text { May, } 25 \text { August, } 24 \text { November }\right) \\
& \left.\left[\ell_{\text {Earth-Sun }}(\phi)\right]_{\text {Min }}=\mathbf{0 . 0 1 6 5 3 8 1 6 8 4 6 3 8 6 7 5} \text { au } \quad \text { (1 January, } 2 \text { April, } 2 \text { July, } 1 \text { October }\right)
\end{aligned}
$$


We have also determined values of time $(t)$ varying semi major axis and semi minor axis vectors $\left[\overrightarrow{\boldsymbol{a}}(t)=\overrightarrow{\boldsymbol{a}}_{\text {Earth-Moon }}(t)\right](55)$ and $\left[\overrightarrow{\boldsymbol{b}}(t)=\overrightarrow{\boldsymbol{b}}_{\text {Earth-Moon }}(t)\right](56)$ of the relative vector ellipse of Earth-Moon system, and their instantaneous focal distance $\left[c(t)=c_{\text {Earth-Moon }}(t)\right](57)$, to an approximation.

$$
\begin{aligned}
\overrightarrow{\boldsymbol{a}}_{\text {Earth-Moon }}(t) \simeq \hat{\boldsymbol{x}}[3,016,536.037 \operatorname{Cos}(0.212768714989863 t)-12,370.477] \mathrm{km} \\
+\hat{\boldsymbol{y}} 3,302,009.781 \operatorname{Sin}(0.212768714989863 t) \mathrm{km} \\
+\hat{\boldsymbol{z}} 1,343,048.374 \operatorname{Cos}(0.212768714989863 t) \mathrm{km} \\
\overrightarrow{\boldsymbol{b}}_{\text {Earth-Moon }}(t)=-\hat{\boldsymbol{x}} 3,016,536.037 \operatorname{Sin}(0.212768714989863 t) \mathrm{km} \\
+\hat{\boldsymbol{y}}[3,302,009.781 \operatorname{Cos}(0.212768714989863 t)-12,370.477] \mathrm{km} \\
-\hat{\boldsymbol{z}} 1,343,048.374 \operatorname{Sin}(0.212768714989863 t) \mathrm{km} \\
c_{\text {Earth-Moon }}(t)=7,062,892,780.525|\operatorname{Cos}(0.212768714989863 t)| \mathrm{km}^{2} \\
(\text { Focal Distance of Earth-Moon system })
\end{aligned}
$$

Note here that in Eq.s (55) - (57), based on our analysis, the time $(t)$ must be measured in [days] from a date that coincides with an Earth to Moon distance maximum in the month of March once in every 3 year cycles of Earth, such as March 18, 2017 and March 24, 2020.

Much more detail on our research results, such as about tilts, wobble angles, libration, and orientation variation cycles of the distance vector between Earth and Moon revolution centers, may be found in our book "Everyhing Is A Circle: A New Model For Orbits Of Bodies In The Universe"1.

\section{AUTHOR CONTRIBUTIONS}

Aslı Pinar Tan is the only author contributing to this Article.

\section{DATA AVAILIBILITY}

Data created and analyzed in this study is shared in the Reference 3 data file. 


\title{
DECLARATION OF INTEREST STATEMENT
}

The Author declares that there is no conflict of interest.

\begin{abstract}
KEYWORDS
Solar System, Planetary System, Planet, Satellite, Sun, Earth, Moon, Topology, Circle, Ellipse, Orbit, Trajectory, Orbital Mechanics
\end{abstract}

\begin{abstract}
ABOUT THE AUTHOR
Aslı Pınar Tan's findings in this Article are part of all her findings as a result of her personal theoretical studies and research over the years independent from any institution or university, which she has published in the book "Everyhing Is A Circle: A New Model For Orbits Of Bodies In The Universe", and will further publish in a set of books and other articles. She is an Electrical \& Electronics Engineer, Bsc. 1995 and Msc. 1997 graduated from Bilkent University, Ankara, Turkey, and she also holds a degree in Multi-Disciplinary Space Studies program graduated in 2000 from International Space University, Strasbourg, France. She has worked professionally more than 20 years in the Global ICT / Telecom industry, in addition to her academic career in Electromagnetics and Space Applications, all independent from this research. She has been granted a Young Scientist Award by URSI (Union Radio Science Internationale) in 1998 based on her Master Thesis Research results presenting a systematic methodology for High-Frequency Radar Antenna Design using the Genetic Algorithm and Equivalent Edge Currents, to generate plain waves in the near field, which is utilized in the industry since then.

(For more: $\underline{\text { http://www.linkedin.com/in/apinartan) }}$
\end{abstract}




\section{REFERENCES}

This article is a summary of the research results that have been published in detail in the following book. These original research results in Turkish are under Notary registration in Istanbul, Turkey where the Author lives.

1. Tan, A.P., Everything is a Circle: A New Model For Orbits of Bodies in the Universe, Amazon Kindle Publishing, ISBN \& EAN 979-8567574669, 2020

Paperback Book: https://www.amazon.com/gp/product/B08NYG14X8

Kindle eBook: https://www.amazon.com/gp/product/B08PVS2FBW

2. Tan, A.P. Distance Between Two Circles In Any Number Of Dimensions Is A Vector Ellipse, Preprints 2021, 2021040632 (doi: 10.20944/preprints202104.0632.v1)

3. File: Tan, A. P., Asli Pinar Tan Analysis Based on Earth-Sun distance (d) Landsat.xlsx, https://www.dropbox.com/scl/fi/th5d3d5ur8d1mb60aitou/Asli-Pinar-Tan-Analysis-Based-onEarth-Sun-distance-d-Landsat.xlsx?dl=0\&rlkey=m3spxqxbxtnumrj2wvtg3312v (2020) 